\title{
Complemented congruences on double Ockham algebras
}

\author{
C. Mendes
}

\begin{abstract}
For $n \in \mathbb{N}$ and $m \in \mathbb{N}_{0}$, an algebra $\mathcal{L}=(L, \wedge, \vee, f, g, 0,1)$ of type $(2,2,1,1,0,0)$ is said to be a double $\mathrm{K}_{n, m}$-algebra, if $\mathcal{L}$ is a double Ockham algebra that satisfies the identities $f^{2 n+m}=f^{m}, g^{2 n+m}=g^{m}, f g=g^{2 z n}$ and $g f=f^{2 z n}$, where $z$ is the smallest natural number greater than or equal to $m / 2 n$. In [2], T. Blyth, A. Noor and J. Varlet study congruences on some double $\mathrm{K}_{1,1}$-algebras. They describe the complement (when it exists) of a principal congruence and, using this description, they also determine when the complement exists. In this paper we generalize this work for double $K_{n, m}$-algebras.
\end{abstract}

\section{Preliminaries}

The variety $\mathbf{O}$ of Ockham algebras is the class of all algebras $(L, \wedge, \vee, h, 0,1)$ of type $(2,2,1,0,0)$ such that $(L, \wedge, \vee, 0,1)$ is a bounded distributive lattice and $h$ is a dual endomorphism of this lattice, i.e., $h(0)=1, h(1)=0$, $h(x \wedge y)=h(x) \vee h(y)$ and $h(x \vee y)=h(x) \wedge h(y)$. These algebras were defined by J. Berman in [1]. We write $(L, h)$ for an Ockham algebra $(L, \wedge, \vee, h, 0,1)$ and we represent both the universe $L$ and the lattice $(L, \wedge, \vee, 0,1)$ by $L$. The subvariety of $\mathbf{O}$ characterized by the identity $h^{2 n+m}=h^{m}, n \in \mathbb{N}$ and $m \in \mathbb{N}_{0}$, is denoted by $\mathbf{K}_{n, m}$ and the elements of this class are called $\mathbf{K}_{n, m}$-algebras. Further information about Ockham algebras and $\mathrm{K}_{n, m}$-algebras can be found in [1] and [3].

For each $\mathcal{L}=(L, h) \in \mathbf{O}$, and for all $n \in \mathbb{N}$ and $m \in \mathbb{N}_{0}$, the sets $h^{m}(L)$ and $L_{n, m}=\left\{x \in L: h^{2 n+m}(x)=h^{m}(x)\right\}$ are subuniverses of $\mathcal{L}$. By $h^{m}(\mathcal{L})$ and $\mathcal{L}_{n, m}$ we denote the subalgebras $\left(h^{m}(L), h\right)$ and $\left(L_{n, m}, h\right)$ of $\mathcal{L}$, respectively. It is useful to notice that, if $\mathcal{L} \in \mathbf{K}_{n, m}$ then $h^{m}(\mathcal{L}) \in \mathbf{K}_{n, 0}$.

Associated to Ockham algebras we have the notion of double Ockham algebras, introduced by M. Sequeira in [5]. A double Ockham algebra is an algebra $\mathcal{L}=(L, \wedge, \vee, f, g, 0,1)$ of type $(2,2,1,1,0,0)$ such that $(L, \wedge, \vee, f, 0,1)$ and $(L, \wedge, \vee, g, 0,1)$ are Ockham algebras. The variety of double Ockham algebras is represented by $\mathbf{O}_{2}$. We denote a double Ockham algebra $\mathcal{L}=(L, \wedge, \vee, f, g, 0,1)$ by $\mathcal{L}=(L, f, g)$ and we represent by $L$, both, the universe $L$ and the distributive lattice $(L, \wedge, \vee, 0,1)$. For the Ockham algebras that are reduct of $\mathcal{L}=(L, f, g)$ we write $(L, f)$ and $(L, g)$.

Let $\mathcal{L}=(L, f, g) \in \mathbf{O}_{2}$. For each $h \in\{f, g\}$, and all $n \in \mathbb{N}$ and all $m \in \mathbb{N}_{0}$, we represent by $L_{m, n}^{h}$ the set $\left\{x \in L: h^{2 n+m}(x)=h^{m}(x)\right\}$. We write $\left(L_{n, m}^{f}, f\right)$ and

2000 Mathematics Subject Classification: 06D30, 06B10, 08A30.

Key words and phrases: Distributive lattices, Ockham algebras, double Ockham algebras, congruences. 
$\left(L_{n, m}^{g}, g\right)$ for the greatest subalgebras of $(L, f)$ and $(L, g)$, respectively, that belong to $\mathbf{K}_{n, m}$.

Let $n, m \in \mathbb{N}$ and let $q$ be the smallest natural number that is greater than or equal to $m / 2 n$; in what follows this element will be denoted by $\lceil m / 2 n\rceil$. The subvariety of $\mathbf{O}_{2}$ characterized by the identities $f^{2 n+m}=f^{m}, g^{2 n+m}=g^{m}$, $g f=f^{2 q n}, f g=g^{2 q n}$ is represented by $\mathbf{D K}_{n, m},[5]$, and the elements of this variety are called double $\mathrm{K}_{n, m}$-algebras.

Given $\mathcal{L}=(L, f, g) \in \mathbf{D} \mathbf{K}_{n, m}$, we have that $f^{m}(L)$ is a subuniverse of $\mathcal{L}$. So, $\left(f^{m}(L), f, g\right)$ is a subalgebra of $\mathcal{L}$, that we denote by $f^{m}(\mathcal{L})$, and the Ockham algebras $\left(f^{m}(L), f\right)$ and $\left(f^{m}(L), g\right)$ are subalgebras of $(L, f)$ and $(L, g)$, respectively.

About double $\mathrm{K}_{n, m}$-algebras it is useful to remind that if $\mathcal{L}=(L, f, g) \in \mathbf{D K}_{n, m}$, then $f^{2 n+k}=f^{k}$ and $g^{2 n+k}=g^{k}$, for all $k \geq m$. We denote by $r(t)$ the remainder of the integer $t$ on division by $2 n$ and, for $1 \leq i, j \leq 2 n+m-1$, let $z_{i, j}=m+r(j-i-m)$. Taking into account the relation between operations $f$ and $g$ it follows that:

Lemma 1.1. [5, Proposition 2] Let $n, m \in \mathbb{N}, \mathcal{L}=(L, f, g) \in \mathbf{D K}_{n, m}$ and $q=\lceil m / 2 n\rceil$. Then

i) $f^{i} g^{i}=g^{q 2 n}, \quad g^{i} f^{i}=f^{q 2 n}, \quad 1 \leq i \leq 2 n+m-1$.

ii) $g^{i} f^{j}=f^{z_{i, j}}, \quad f^{j} g^{i}=g^{z_{j, i}}, \quad 1 \leq i, j \leq 2 n+m-1$.

iii) $f^{m}(L)=g^{m}(L)$.

We now present some notation related to congruences. Given an algebra $\mathcal{L}$ (element of $\mathbf{O}$ or element of $\mathbf{O}_{2}$ ) we denote by:

- $\operatorname{Con}_{\text {lat }} \mathcal{L}$ and Con $\mathcal{L}$, the congruence lattice of the distributive lattice $L$ (reduct of $\mathcal{L})$ and the algebra $\mathcal{L}$, respectively;

- $\theta_{\text {lat }}(a, b)$ and $\theta(a, b)$ the least congruence of $\operatorname{Con}_{\text {lat }} \mathcal{L}$ and Con $\mathcal{L}$, respectively, that identifies the elements $a$ and $b$ of $L$;

- $\mathbf{0}$ and $\mathbf{1}$ the identity and the universal congruence of $\mathcal{L}$, respectively;

- $\theta_{L^{\prime}}$ a congruence defined on a subalgebra $\mathcal{L}^{\prime}$ of $\mathcal{L}\left(\mathbf{0}_{L^{\prime}}\right.$ and $\mathbf{1}_{L^{\prime}}$ represent, respectively, the identity and the universal congruences of $\left.\mathcal{L}^{\prime}\right)$.

For $\mathcal{L}=(L, f, g) \in \mathbf{D K}_{n, m}$ we represent by:

- $\operatorname{Con}_{f} \mathcal{L}$ and $\operatorname{Con}_{g} \mathcal{L}$, the congruence lattice of the algebra $(L, f)$ and the algebra $(L, g)$, respectively;

- $\theta_{f}(a, b)$ and $\theta_{g}(a, b)$ the least congruence of $\operatorname{Con}_{f} \mathcal{L}$ and $\operatorname{Con}_{g} \mathcal{L}$, respectively, that identifies the elements $a$ and $b$ of $L$;

- $\theta_{f, f^{m}(L)}(a, b), \theta_{g, f^{m}(L)}(a, b)$ the least congruence of $\operatorname{Con}_{f} f^{m}(\mathcal{L})$ and $\operatorname{Con}_{g} f^{m}(\mathcal{L})$, respectively, that identifies the elements $a$ and $b$ of $f^{m}(L)$. 
Remark: Let $\mathcal{L}=(L, f, g) \in \mathbf{D K}_{n, m}$. Given $\theta_{f} \in \operatorname{Con}_{f} \mathcal{L}$ and $\theta_{g} \in \operatorname{Con}_{g} \mathcal{L}$, since $\theta_{f}, \theta_{g} \in \mathrm{Con}_{\text {lat }} \mathcal{L}$, we represent by $\theta_{f} \vee \theta_{g}$ and $\theta_{f} \wedge \theta_{g}$, respectively, the join and the meet of $\theta_{f}$ and $\theta_{g}$ on $\operatorname{Con}_{\text {lat }} \mathcal{L}$.

To study principal congruences of $\mathcal{L}=(L, f, g) \in \mathbf{O}_{2}$ it suffices to consider the congruence $\theta(a, b)$ for $a \leq b$ since, for any congruence $\theta$ of a lattice $L^{\prime}$ and any $x, y \in L^{\prime}$, we have $(x, y) \in \theta$ if and only if $(x \wedge y, x \vee y) \in \theta$.

For any $\mathcal{L} \in \mathbf{O}$ (resp. $\mathbf{O}_{2}$ ), the lattice $\operatorname{Con} \mathcal{L}$ is distributive. Also, for any subalgebra $\mathcal{L}^{\prime}$ of an algebra $\mathcal{L} \in \mathbf{O}$, each congruence defined on $\mathcal{L}^{\prime}$ is the restriction of some congruence defined on $\mathcal{L}$. This means that the variety $\mathbf{O}$ satisfies the congruence extension property. Consequently we have the following:

Lemma 1.2. If $\mathcal{L} \in \mathbf{O}, \mathcal{L}^{\prime}$ is a subalgebra of $\mathcal{L}$ and $a, b \in L^{\prime}$, then

$$
\left.\theta(a, b)\right|_{L^{\prime}}=\theta_{L^{\prime}}(a, b) .
$$

The following result, that establishes that any principal congruence on $\mathcal{L} \in \mathbf{K}_{n, m}$ is the join of principal congruences on the distributive lattice $L$, is fundamental in the investigation of congruences defined on $\mathrm{K}_{n, m}$-algebras.

Lemma 1.3. [1, Corollary Theorem 1] If $\mathcal{L}=(L, h) \in \mathbf{K}_{n, m}$ and $a, b \in L$ with $a \leq b$ then

$$
\theta(a, b)=\bigvee_{i=0}^{2 n+m-1} \theta_{\mathrm{lat}}\left(h^{i}(a), h^{i}(b)\right) .
$$

For double $\mathrm{K}_{n, m}$-algebras it is also possible to establish a result similar to this one:

Lemma 1.4. [5] If $\mathcal{L}=(L, f, g) \in \mathbf{D K}_{n, m}$ and $a, b \in L$ with $a \leq b$, then

$$
\theta(a, b)=\theta_{\mathrm{lat}}(a, b) \vee \bigvee_{i=1}^{2 n+m-1} \theta_{\mathrm{lat}}\left(f^{i}(a), f^{i}(b)\right) \vee \bigvee_{j=1}^{2 n+m-1} \theta_{\mathrm{lat}}\left(g^{j}(a), g^{j}(b)\right) .
$$

From Lemmas 1.3 and 1.4 is immediate that:

Lemma 1.5. If $\mathcal{L}=(L, f, g) \in \mathbf{D K}_{n, m}$ and $a, b \in L$ are such that $a \leq b$, then

$$
\theta(a, b)=\theta_{f}(a, b) \vee \theta_{g}(a, b) .
$$

Definition 1.6. By a $p$-ladder in an ordered set $E$ we shall mean a subset of $E$ that consists of two $p$-chains $a_{1}<\ldots<a_{p}$ and $b_{1}<\ldots<b_{p}$ such that $a_{i} \leq b_{i}$ for $i=1, \ldots, p$. We shall denote a $p$-ladder by $\left(a_{i}, b_{i}\right)_{p}$.

Let $T=\{0,1, \ldots, n-1\}$ and, for $s \in\{1, \ldots, n\}$, let $T_{s}=\{J: J \subseteq T,|J|=s\}$. Let $\mathcal{L}=(L, h) \in \mathbf{K}_{n, m}$ and $a, b \in L$ be such that $a \leq b$. For $s \in\{1, \ldots, n\}$, let

$$
\widetilde{a}_{h, s}=\bigwedge_{J \in T_{s}} \bigvee_{j \in J} h^{2 j}(a), \quad \widetilde{b}_{h, s}=\bigwedge_{J \in T_{s}} \bigvee_{j \in J} h^{2 j}(b)
$$


It is easy to prove that the set $\left\{\widetilde{a}_{h, s}, \widetilde{b}_{h, s}: s=1, \ldots, n\right\}$ is an $n$-ladder consisting of elements that belong to the subalgebra $\mathcal{L}_{1, m}$. In the following theorem, which is an unpublished result of M. Sequeira, this $n$-ladder is used to establish that any principal congruence defined on a double $\mathrm{K}_{n, m}$-algebra $\mathcal{L}=(L, f, g)$ is the join of principal congruences generated by elements of $L_{1, m}$.

Theorem 1.7. Let $\mathcal{L}=(L, h) \in \mathbf{K}_{n, m}$ and $a, b \in L$ be such that $a \leq b$. Then

$$
\theta(a, b)=\bigvee_{s=1}^{n} \theta\left(\widetilde{a}_{h, s}, \widetilde{b}_{h, s}\right)
$$

Next Lemma follows immediately from Theorem 1.7 and Lemma 1.5 and describes each principal congruence defined on a double $\mathrm{K}_{n, m}$-algebra $\mathcal{L}=(L, f, g)$ by means of elements of $L_{1, m}^{f}$ and elements of $L_{1, m}^{g}$.

Lemma 1.8. If $\mathcal{L}=(L, f, g) \in \mathbf{D K}_{n, m}$ and $a, b \in L$ with $a \leq b$, then

$$
\theta(a, b)=\bigvee_{s=1}^{n} \theta_{f}\left(\widetilde{a}_{f, s}, \widetilde{b}_{f, s}\right) \vee \bigvee_{t=1}^{n} \theta_{g}\left(\widetilde{a}_{g, t}, \widetilde{b}_{g, t}\right)
$$

The purpose of this paper is to characterize the principal congruences $\theta(a, b)$ on double $\mathrm{K}_{n, m}$-algebras that are complemented. The study of these congruences is strongly related to the following theorem which establishes that, given $\mathcal{L}=(L, f) \in \mathbf{O}$, all congruences generated by elements of $L_{1,0}$ are complemented. This theorem is, also, an unpublished result of M. Sequeira [5].

Theorem 1.9. If $\mathcal{L}=(L, h) \in \mathbf{O}$ and $a, b \in L_{1,0}$ with $a \leq b$, then $\theta(a, b)$ is complemented in $\operatorname{Con}(\mathcal{L})$, and

$$
\begin{aligned}
\theta(a, b)^{\prime} & =\theta(h(a) \vee b, 1) \vee \theta(h(a), h(a) \vee a) \vee \theta(b, b \vee h(b)) \\
& =\theta(0, a \wedge h(b)) \vee \theta(a \wedge h(a), a) \vee \theta(b \wedge h(b), h(b)) .
\end{aligned}
$$

\section{Congruences}

Let $\mathcal{L}=(L, f, g) \in \mathbf{D K}_{n, m}$ and $a, b \in L$ be such that $a \leq b$. By Lemma 1.5 , the congruence $\theta(a, b)$ is the join, on $\operatorname{Con}_{\text {lat }} \mathcal{L}$, of a principal congruence on $(L, f)$ and a principal congruence on $(L, g)$. So, it is natural that the study of $\theta(a, b)$ uses various results obtained on [4]; where the author studies complemented congruences on $\mathrm{K}_{n, m}$-algebras. Moreover, similar results for double $\mathrm{K}_{n, m}$ algebras, involving the relation between the operations $f$ and $g$, need to be established. We start this section establishing and proving that results.

Lemma 2.1. Let $\mathcal{L}=(L, f, g) \in \mathbf{D K}_{n, m}, i \in \mathbb{N}, k \in \mathbb{N}$ be such that $k \geq m$ and $a, b \in L$ with $a \leq b$. Then, given $x, y \in L$

$$
(x, y) \in \theta_{\text {lat }}\left(g^{i}(a), g^{i}(b)\right) \Rightarrow\left(f^{k}(x), f^{k}(y)\right) \in \theta_{\text {lat }}\left(g^{t}(a), g^{t}(b)\right),
$$

for some $t \in\{m, \ldots, 2 n+m-1\}$. 
Proof. Let $x, y \in L . \quad$ If $(x, y) \in \theta_{\text {lat }}\left(g^{i}(a), g^{i}(b)\right)$, for some $i \in \mathbb{N}$, then $\left(f^{k}(x), f^{k}(y)\right) \in \theta_{\text {lat }}\left(f^{k}\left(g^{i}(a)\right), f^{k}\left(g^{i}(b)\right)\right)$. From Lemma 1.1 it follows that $f^{k}\left(g^{i}(a)\right)=g^{t}(a)$ and $f^{k}\left(g^{i}(b)\right)=g^{t}(b)$, with $t \in\{m, \ldots, 2 n+m-1\}$.

Lemma 2.2. Let $\mathcal{L}=(L, f, g) \in \mathbf{D K}_{n, m}$ and $a, b \in L$ with $a \leq b$. Then

$$
\left.\theta_{g}(a, b)\right|_{f^{m}(L)}=\left.\bigvee_{k=0}^{2 n+m-1} \theta_{\text {lat }}\left(g^{k}(a), g^{k}(b)\right)\right|_{f^{m}(L)} .
$$

Proof. The result follows immediately from [4, Lemma 2.3] since $f^{m}(L)=g^{m}(L)$, $\theta_{g}(a, b) \in \operatorname{Con}_{g} \mathcal{L}$ and $(L, g) \in \mathbf{K}_{n, m}$.

Lemma 2.3. Let $\mathcal{L}=(L, f, g) \in \mathbf{D K}_{n, m}$ and $a, b \in L$ be such that $a \leq b$. Then,

$$
\begin{aligned}
\left.\theta(a, b)\right|_{f^{m}(L)}=\left.\theta_{\text {lat }}(a, b)\right|_{f^{m}(L)} & \left.\vee \bigvee_{i=1}^{2 n+m-1} \theta_{\text {lat }}\left(f^{i}(a), f^{i}(b)\right)\right|_{f^{m}(L)} \\
& \left.\vee \bigvee_{j=1}^{2 n+m-1} \theta_{\text {lat }}\left(g^{j}(a), g^{j}(b)\right)\right|_{f^{m}(L)} .
\end{aligned}
$$

Proof. By Lemma 1.4 we have

$$
\theta(a, b)=\theta_{\text {lat }}(a, b) \vee \bigvee_{i=1}^{2 n+m-1} \theta_{\text {lat }}\left(f^{i}(a), f^{i}(b)\right) \vee \bigvee_{j=1}^{2 n+m-1} \theta_{\text {lat }}\left(g^{j}(a), g^{j}(b)\right)
$$

and it is obvious that

$$
\begin{aligned}
& \left.\left.\left.\theta_{\text {lat }}(a, b)\right|_{f^{m}(L)} \vee \bigvee_{i=1}^{2 n+m-1} \theta_{\text {lat }}\left(f^{i}(a), f^{i}(b)\right)\right|_{f^{m}(L)} \vee \bigvee_{j=1}^{2 n+m-1} \theta_{\text {lat }}\left(g^{j}(a), g^{j}(b)\right)\right|_{f^{m}(L)} \\
& \leq\left.\left[\theta_{\text {lat }}(a, b) \vee \bigvee_{i=1}^{2 n+m-1} \theta_{\text {lat }}\left(f^{i}(a), f^{i}(b)\right) \vee \bigvee_{j=1}^{2 n+m-1} \theta_{\text {lat }}\left(g^{j}(a), g^{j}(b)\right)\right]\right|_{f^{m}(L)} .
\end{aligned}
$$

Let $x, y$ be elements of $L$ such that $\left.(x, y) \in \theta(a, b)\right|_{f^{m}(L)}$, i.e., such that

$$
\left.(x, y) \in\left[\theta_{\text {lat }}(a, b) \vee \bigvee_{i=1}^{2 n+m-1} \theta_{\text {lat }}\left(f^{i}(a), f^{i}(b)\right) \vee \bigvee_{j=1}^{2 n+m-1} \theta_{\text {lat }}\left(g^{j}(a), g^{j}(b)\right)\right]\right|_{f^{m}(L)} .
$$

Then $x, y \in f^{m}(L)$ and there exist $s \in \mathbb{N}$ and $x_{0}=x, x_{1}, \ldots, x_{s}=y \in L$ such that, for all $v \in\{0, \ldots, s-1\}$,

$$
\begin{aligned}
& \text { - }\left(x_{v}, x_{v+1}\right) \in \theta_{\text {lat }}\left(f^{i_{v}}(a), f^{i_{v}}(b)\right), \text { for some } i_{v} \in\{0, \ldots, 2 n+m-1\} \\
& \text { or } \\
& \text { - }\left(x_{v}, x_{v+1}\right) \in \theta_{\text {lat }}\left(g^{j_{v}}(a), g^{j_{v}}(b)\right), \text { for some } j_{v} \in\{1, \ldots, 2 n+m-1\} .
\end{aligned}
$$

In what follows we consider $q=\lceil m / 2 n\rceil$. Thus, if $\left(x_{v}, x_{v+1}\right) \in \theta_{\text {lat }}\left(f^{i_{v}}(a), f^{i_{v}}(b)\right)$ we have by [4, Lemma 2.2] that $\left(f^{22 n}\left(x_{v}\right), f^{q 2 n}\left(x_{v+1}\right)\right) \in \theta_{\text {lat }}\left(f^{t_{v}}(a), f^{t_{v}}(b)\right)$, for some $t_{v} \in\{m, \ldots, 2 n+m-1\}$. Since $f^{q 2 n}\left(x_{v}\right), f^{q 2 n}\left(x_{v+1}\right)$ are elements of $f^{m}(L)$, then $\left.\left(f^{q 2 n}\left(x_{v}\right), f^{q 2 n}\left(x_{v+1}\right)\right) \in \theta_{\text {lat }}\left(f^{t_{v}}(a), f^{t_{v}}(b)\right)\right|_{f^{m}(L)}$. 
If $\left(x_{v}, x_{v+1}\right) \in \theta_{\text {lat }}\left(g^{j_{v}}(a), g^{j_{v}}(b)\right)$ it is also possible to conclude, in this case using Lemma 2.1, that $\left.\left(f^{q 2 n}\left(x_{v}\right), f^{q 2 n}\left(x_{v+1}\right)\right) \in \theta_{\text {lat }}\left(g^{s_{v}}(a), g^{s_{v}}(b)\right)\right|_{f^{m}(L)}$, for some $s_{v} \in\{m, \ldots, 2 n+m-1\}$.

Consequently

$$
\begin{aligned}
\left.\left(f^{q 2 n}(x), f^{q 2 n}(y)\right) \in \theta_{\mathrm{lat}}(a, b)\right|_{f^{m}(L)} & \left.\vee \bigvee_{i=1}^{2 n+m-1} \theta_{\text {lat }}\left(f^{i}(a), f^{i}(b)\right)\right|_{f^{m}(L)} \\
& \left.\vee \bigvee_{j=1}^{2 n+m-1} \theta_{\text {lat }}\left(g^{j}(a), g^{j}(b)\right)\right|_{f^{m}(L)}
\end{aligned}
$$

where $f^{q 2 n}(x)=x$ and $f^{q 2 n}(y)=y$ since $x, y \in f^{m}(L)$. Thus we have

$$
\begin{aligned}
\left.\theta(a, b)\right|_{f^{m}(L)} \leq\left.\theta_{\text {lat }}(a, b)\right|_{f^{m}(L)} & \left.\vee \bigvee_{i=1}^{2 n+m-1} \theta_{\text {lat }}\left(f^{i}(a), f^{i}(b)\right)\right|_{f^{m}(L)} \\
& \left.\vee \bigvee_{j=1}^{2 n+m-1} \theta_{\text {lat }}\left(g^{j}(a), g^{j}(b)\right)\right|_{f^{m}(L)} .
\end{aligned}
$$

This lemma is used to prove the following result:

Lemma 2.4. Let $\mathcal{L}=(L, f, g) \in \mathbf{D K}_{n, m}$ and $a, b \in L$ be such that $a \leq b$. Then,

$$
\left.\theta(a, b)\right|_{f^{m}(L)}=\left.\left.\bigvee_{s=1}^{n} \theta_{f}\left(\widetilde{a}_{f, s}, \widetilde{b}_{f, s}\right)\right|_{f^{m}(L)} \vee \bigvee_{t=1}^{n} \theta_{g}\left(\widetilde{a}_{g, t}, \widetilde{b}_{g, t}\right)\right|_{f^{m}(L)}
$$

Proof. By Lemma 2.3 we have

$$
\begin{aligned}
\left.\theta(a, b)\right|_{f^{m}(L)}=\left.\theta_{\text {lat }}(a, b)\right|_{f^{m}(L)} & \left.\vee \bigvee_{i=1}^{2 n+m-1} \theta_{\text {lat }}\left(f^{i}(a), f^{i}(b)\right)\right|_{f^{m}(L)} \\
& \left.\vee \bigvee_{j=1}^{2 n+m-1} \theta_{\text {lat }}\left(g^{j}(a), g^{j}(b)\right)\right|_{f^{m}(L)} .
\end{aligned}
$$

From [4, Lemma 2.3] and Lemma 2.2 it follows that

$$
\left.\theta(a, b)\right|_{f^{m}(L)}=\left.\left.\theta_{f}(a, b)\right|_{f^{m}(L)} \vee \theta_{g}(a, b)\right|_{f^{m}(L)}
$$

and, by Theorem 1.7

$$
\left.\theta(a, b)\right|_{f^{m}(L)}=\left.\left.\left(\bigvee_{s=1}^{n} \theta_{f}\left(\widetilde{a}_{f, s}, \widetilde{b}_{f, s}\right)\right)\right|_{f^{m}(L)} \vee\left(\bigvee_{t=1}^{n} \theta_{g}\left(\widetilde{a}_{g, t}, \widetilde{b}_{g, t}\right)\right)\right|_{f^{m}(L)}
$$

Finally, using [4, Lemma 2.4] and since $f^{m}(L)=g^{m}(L)$, we have

$$
\left.\theta(a, b)\right|_{f^{m}(L)}=\left.\left.\bigvee_{s=1}^{n} \theta_{f}\left(\widetilde{a}_{f, s}, \widetilde{b}_{f, s}\right)\right|_{f^{m}(L)} \vee \bigvee_{t=1}^{n} \theta_{g}\left(\widetilde{a}_{g, t}, \widetilde{b}_{g, t}\right)\right|_{f^{m}(L)}
$$

Given an algebra $\mathcal{L} \in \mathbf{O}$ (resp. $\mathcal{L} \in \mathbf{O}_{2}$ ), let $\operatorname{Con}^{\prime} \mathcal{L}$ represent the lattice of complemented congruences on $\mathcal{L}$.

Lemma 2.5. Let $\mathcal{L}=(L, f, g) \in \mathbf{D K}_{n, m}$ and $\theta \in \operatorname{Con} \mathcal{L}$. If $\theta \in \operatorname{Con}^{\prime} \mathcal{L}$, then $\left.\theta\right|_{f^{m}(L)} \in \operatorname{Con}^{\prime} f^{m}(\mathcal{L})$. In fact, if $\theta^{\prime}$ is the complement of $\theta$ in $\operatorname{Con} \mathcal{L}$, then $\left.\theta^{\prime}\right|_{f^{m}(L)}$ is the complement of $\left.\theta\right|_{f^{m}(L)}$ in $\operatorname{Con} f^{m}(\mathcal{L})$. 
Proof. Let $\theta \in \operatorname{Con}^{\prime} \mathcal{L}$ and $\theta^{\prime}$ be the complement of $\theta$ in $\operatorname{Con} \mathcal{L}$. Then $\left.\theta\right|_{f^{m}(L)}$ and $\left.\theta^{\prime}\right|_{f^{m}(L)}$ are elements of $\operatorname{Con} f^{m}(\mathcal{L})$. Since $\theta, \theta^{\prime} \in \operatorname{Con}_{f} \mathcal{L}, \theta^{\prime}$ is also the complement of $\theta$ in $\operatorname{Con}_{f} \mathcal{L}$. By [4, Lemma 2.5] we have that $\left.\theta^{\prime}\right|_{f^{m}(L)}$ is the complement of $\left.\theta\right|_{f^{m}(L)}$ in $\operatorname{Con}_{f} f^{m}(\mathcal{L})$ and, consequently, in $\operatorname{Con} f^{m}(\mathcal{L})$.

Lemma 2.6. Let $\mathcal{L}=(L, f, g) \in \mathbf{D K}_{n, m}$ and $a, b \in L$ with $a \leq b$ and $k \in \mathbb{N}$ be such that $k \geq m$. Then

i) $\theta\left(f^{k}(a), f^{k}(b)\right)=\theta_{f}\left(f^{k}(a), f^{k}(b)\right)$,

ii) $\theta\left(g^{k}(a), g^{k}(b)\right)=\theta_{f}\left(g^{k}(a), g^{k}(b)\right)$,

iii) $\theta\left(g^{k}(a), g^{k}(b)\right)=\theta_{g}\left(g^{k}(a), g^{k}(b)\right)$,

iv) $\theta\left(f^{k}(a), f^{k}(b)\right)=\theta_{g}\left(f^{k}(a), f^{k}(b)\right)$.

Proof. i) By Lemma 1.4 we have

$$
\begin{aligned}
\theta\left(f^{k}(a), f^{k}(b)\right)=\theta_{\text {lat }}\left(f^{k}(a), f^{k}(b)\right) & \vee \bigvee_{i=1}^{2 n+m-1} \theta_{\text {lat }}\left(f^{i}\left(f^{k}(a)\right), f^{i}\left(f^{k}(b)\right)\right) \\
& \vee \bigvee_{j=1}^{2 n+m-1} \theta_{\text {lat }}\left(g^{j}\left(f^{k}(a)\right), g^{j}\left(f^{k}(b)\right)\right) .
\end{aligned}
$$

Since $k=m+r$, for some $r \in \mathbb{N}_{0}$, it follows by Lemma 1.1 that, for all $x \in L$ and $j \in\{1, \ldots, 2 n+m-1\}$,

$$
\begin{aligned}
g^{j}\left(f^{k}(x)\right) & =g^{j}\left(f^{m}\left(f^{r}(x)\right)\right)=f^{z_{j, m}}\left(f^{r}(x)\right) \\
& =f^{z_{j, m}-m}\left(f^{m}\left(f^{r}(x)\right)\right)=f^{z_{j, m}-m}\left(f^{k}(x)\right),
\end{aligned}
$$

with $z_{j, m}-m \in\{0, \ldots, 2 n-1\}$. Thus we have

$$
\theta\left(f^{k}(a), f^{k}(b)\right)=\theta_{\text {lat }}\left(f^{k}(a), f^{k}(b)\right) \vee \bigvee_{i=1}^{2 n+m-1} \theta_{\text {lat }}\left(f^{i}\left(f^{k}(a)\right), f^{i}\left(f^{k}(b)\right)\right)
$$

and by Lemma 1.3 we conclude that $\theta\left(f^{k}(a), f^{k}(b)\right)=\theta_{f}\left(f^{k}(a), f^{k}(b)\right)$; so i) follows. Since $f^{m}(L)=g^{m}(L)$ we have $g^{k}(a)=f^{m}(x)$ and $g^{k}(b)=f^{m}(y)$, for some $x, y \in L$. So case ii) is immediate from i). The proof of iii) is analogous to the one for case i). Case iv) follows from iii).

Definition 2.7. By a $m$-pair, $m \in \mathbb{N}$, we shall mean the ordered pair $(k, l)$ such that

$$
(k, l)= \begin{cases}(m, m+1) & \text { if } m \text { is even; } \\ (m+1, m) & \text { if } m \text { is odd }\end{cases}
$$

It is useful to notice that, if $(k, l)$ is a $m$-pair then $k$ is always even, and $l$ is always odd.

In what follows we consider $\mathcal{L}=(L, f, g) \in \mathbf{D K}_{n, m}$ and, for $z \in L$, $T=\{0,1, \ldots, n-1\}, s \in T$ and $i \in \mathbb{N}$, we represent the elements $f^{i}\left(\widetilde{z}_{f, s}\right)$ and $g^{i}\left(\widetilde{z}_{g, s}\right)$ by $f^{i}\left(\widetilde{z}_{s}\right)$ and $g^{i}\left(\widetilde{z}_{s}\right)$, respectively. Note that we never use the elements $f^{i}\left(\widetilde{z}_{g, s}\right)$ and $g^{i}\left(\widetilde{z}_{f, s}\right)$. Moreover, we denote by $(k, l)$ an $m$-pair. 
Let $a, b \in L$ be such that $a \leq b$ and suppose that $\theta(a, b)$ is complemented. As we will see, the description of the complement of $\theta(a, b)$ is, in fact, related to Theorem 1.9 .

If we take $q=\lceil m / 2 n\rceil$, then $f^{q 2 n}\left(\widetilde{a}_{s}\right), f^{q 2 n}\left(\widetilde{b}_{s}\right) \in f^{m}(L)$ and $g^{q 2 n}\left(\widetilde{a}_{s}\right)$, $g^{q 2 n}\left(\widetilde{b}_{s}\right) \in g^{m}(L)$. Consequently, taking into account that $f^{m}(L)=g^{m}(L)$, it follows by Lemma 2.4, [4, Lemma 2.1] and Lemma 1.2 that:

$$
\begin{aligned}
\left.\theta(a, b)\right|_{f^{m}(L)} & =\left.\left.\bigvee_{s=1}^{n} \theta_{f}\left(\widetilde{a}_{f, s} \widetilde{b}_{f, s}\right)\right|_{f^{m}(L)} \vee \bigvee_{t=1}^{n} \theta_{g}\left(\widetilde{a}_{g, t} \widetilde{b}_{g, t}\right)\right|_{f^{m}(L)} \\
& =\left.\left.\bigvee_{s=1}^{n} \theta_{f}\left(f^{q 2 n}\left(\widetilde{a}_{s}\right), f^{q 2 n}\left(\widetilde{b}_{s}\right)\right)\right|_{f^{m}(L)} \vee \bigvee_{t=1}^{n} \theta_{g}\left(g^{q 2 n}\left(\widetilde{a}_{t}\right), g^{q 2 n}\left(\widetilde{b}_{t}\right)\right)\right|_{f^{m}(L)} \\
& =\bigvee_{s=1}^{n} \theta_{f, f^{m}(L)}\left(f^{q 2 n}\left(\widetilde{a}_{s}\right), f^{q 2 n}\left(\widetilde{b}_{s}\right)\right) \vee \bigvee_{t=1}^{n} \theta_{g, f^{m}(L)}\left(g^{q 2 n}\left(\widetilde{a}_{t}\right), g^{q 2 n}\left(\widetilde{b}_{t}\right)\right) .
\end{aligned}
$$

Since $\widetilde{a}_{f, s}, \widetilde{b}_{f, s} \in L_{1, m}^{f}, \widetilde{a}_{g, t}, \widetilde{b}_{g, t} \in L_{1, m}^{g}$ and $q 2 n \geq m$, then $f^{q 2 n}\left(\widetilde{a}_{s}\right)$, $f^{q 2 n}\left(\widetilde{b}_{s}\right) \in L_{1,0}^{f}$, and $g^{q 2 n}\left(\widetilde{a}_{t}\right), g^{q 2 n}\left(\widetilde{b}_{t}\right) \in L_{1,0}^{g}$. So, by Theorem 1.9 , the congruences $\theta_{f, f^{m}(L)}\left(f^{q 2 n}\left(\widetilde{a}_{s}\right), f^{q 2 n}\left(\widetilde{b}_{s}\right)\right)$ and $\theta_{g, f^{m}(L)}\left(g^{q 2 n}\left(\widetilde{a}_{t}\right), g^{q 2 n}\left(\widetilde{b}_{t}\right)\right)$ are complemented, respectively, in $\operatorname{Con}_{f} f^{m}(\mathcal{L})$ and $\operatorname{Con}_{g} f^{m}(\mathcal{L})$.

Using Lemma 1.2 and [4, Lemma 2.4] it is proved in [4] that

$$
\begin{aligned}
& \theta_{f, f^{m}(L)}\left(f^{q 2 n}\left(\widetilde{a}_{s}\right), f^{q 2 n}\left(\widetilde{b}_{s}\right)\right)^{\prime}=\left.\varphi_{f}\left(\widetilde{a}_{f, s}, \widetilde{b}_{f, s}\right)\right|_{f^{m}(L)} \quad \text { and } \\
& \theta_{g, f^{m}(L)}\left(g^{q 2 n}\left(\widetilde{a}_{t}\right), g^{q 2 n}\left(\widetilde{b}_{t}\right)\right)^{\prime}=\left.\varphi_{g}\left(\widetilde{a}_{g, t}, \widetilde{b}_{g, t}\right)\right|_{f^{m}(L)}
\end{aligned}
$$

where

$$
\begin{aligned}
\varphi_{f}\left(\widetilde{a}_{f, s}, \widetilde{b}_{f, s}\right)=\theta_{f}\left(f^{k}\left(\widetilde{b}_{s}\right) \vee f^{l}\left(\widetilde{a}_{s}\right), 1\right) & \vee \theta_{f}\left(f^{k}\left(\widetilde{b}_{s}\right), f^{k}\left(\widetilde{b}_{s}\right) \vee f^{l}\left(\widetilde{b}_{s}\right)\right) \\
& \vee \theta_{f}\left(f^{l}\left(\widetilde{a}_{s}\right), f^{l}\left(\widetilde{a}_{s}\right) \vee f^{k}\left(\widetilde{a}_{s}\right)\right) \quad \text { and } \\
\varphi_{g}\left(\widetilde{a}_{g, t}, \widetilde{b}_{g, t}\right)=\theta_{g}\left(g^{k}\left(\widetilde{b}_{t}\right) \vee g^{l}\left(\widetilde{a}_{t}\right), 1\right) & \vee \theta_{g}\left(g^{k}\left(\widetilde{b}_{t}\right), g^{k}\left(\widetilde{b}_{t}\right) \vee g^{l}\left(\widetilde{b}_{t}\right)\right) \\
& \vee \theta_{g}\left(g^{l}\left(\widetilde{a}_{t}\right), g^{l}\left(\widetilde{a}_{t}\right) \vee g^{k}\left(\widetilde{a}_{t}\right)\right)
\end{aligned}
$$

Since $k \geq m$ and $l \geq m$, it follows by Lemma 2.6 that each congruence $\theta_{f}\left(f^{k}\left(\widetilde{b}_{s}\right) \vee f^{l}\left(\widetilde{a}_{s}\right), 1\right), \theta_{f}\left(f^{k}\left(\widetilde{b}_{s}\right), f^{k}\left(\widetilde{b}_{s}\right) \vee f^{l}\left(\widetilde{b}_{s}\right)\right)$ and $\theta_{g}\left(g^{l}\left(\widetilde{a}_{t}\right), g^{l}\left(\widetilde{a}_{t}\right) \vee g^{k}\left(\widetilde{a}_{t}\right)\right)$ is an element of Con $\mathcal{L}$; so $\varphi_{f}\left(\widetilde{a}_{f, s}, \widetilde{b}_{f, s}\right)$ is an element of Con $\mathcal{L}$. Now, taking into account that $f^{m}(L)$ is a subuniverse of $\mathcal{L}$ we conclude that $\left.\varphi_{f}\left(\widetilde{a}_{f, s}, \widetilde{b}_{f, s}\right)\right|_{f^{m}(L)} \in \operatorname{Con} f^{m}(\mathcal{L})$. In a similar way we prove that $\left.\varphi_{g}\left(\widetilde{a}_{g, t}, \widetilde{b}_{g, t}\right)\right|_{f^{m}(L)} \in \operatorname{Con} f^{m}(\mathcal{L})$ and, also by Lemma 2.6, we have $\theta_{f, f^{m}(L)}\left(f^{q 2 n}\left(\widetilde{a}_{s}\right), f^{q 2 n}\left(\widetilde{b}_{s}\right)\right), \theta_{g, f^{m}(L)}\left(g^{q 2 n}\left(\widetilde{a}_{t}\right), g^{q 2 n}\left(\widetilde{b}_{t}\right)\right) \in \operatorname{Con} f^{m}(\mathcal{L})$. So, both congruences $\theta_{f, f^{m}(L)}\left(f^{q 2 n}\left(\widetilde{a}_{s}\right), f^{q 2 n}\left(\widetilde{b}_{s}\right)\right)$ and $\theta_{g, f^{m}(L)}\left(g^{q 2 n}\left(\widetilde{a}_{t}\right), g^{q 2 n}\left(\widetilde{b}_{t}\right)\right)$ are complemented in $\operatorname{Con} f^{m}(\mathcal{L})$. Since

$$
\left.\theta(a, b)\right|_{f^{m}(L)}=\bigvee_{s=1}^{n} \theta_{f, f^{m}(L)}\left(f^{q 2 n}\left(\widetilde{a}_{s}\right), f^{q 2 n}\left(\widetilde{b}_{s}\right)\right) \vee \bigvee_{t=1}^{n} \theta_{g, f^{m}(L)}\left(g^{q 2 n}\left(\widetilde{a}_{t}\right), g^{q 2 n}\left(\widetilde{b}_{t}\right)\right)
$$


it is obvious that $\left.\theta(a, b)\right|_{f^{m}(L)}$ is also complemented in $\operatorname{Con} f^{m}(\mathcal{L})$ and we have:

$$
\begin{aligned}
\left(\left.\theta(a, b)\right|_{f^{m}(L)}\right)^{\prime} & =\bigwedge_{s=1}^{n} \theta_{f, f^{m}(L)}\left(f^{q 2 n}\left(\widetilde{a}_{s}\right), f^{q 2 n}\left(\widetilde{b}_{s}\right)\right)^{\prime} \wedge \bigwedge_{t=1}^{n} \theta_{g, f^{m}(L)}\left(g^{q 2 n}\left(\widetilde{a}_{t}\right), g^{q 2 n}\left(\widetilde{b}_{t}\right)\right)^{\prime} \\
& =\left(\left.\bigwedge_{s=1}^{n} \varphi_{f}\left(\widetilde{a}_{f, s}, \widetilde{b}_{f, s}\right)\right|_{f^{m}(L)}\right) \wedge\left(\left.\bigwedge_{t=1}^{n} \varphi_{g}\left(\widetilde{a}_{g, t}, \widetilde{b}_{g, t}\right)\right|_{f^{m}(L)}\right) \\
& =\left.\left(\bigwedge_{s=1}^{n} \varphi_{f}\left(\widetilde{a}_{f, s}, \widetilde{b}_{f, s}\right) \wedge \bigwedge_{t=1}^{n} \varphi_{g}\left(\widetilde{a}_{g, t}, \widetilde{b}_{g, t}\right)\right)\right|_{f^{m}(L)} .
\end{aligned}
$$

Let $\varphi_{f, g}$ stand for $\bigwedge_{s=1}^{n} \varphi_{f}\left(\widetilde{a}_{f, s}, \widetilde{b}_{f, s}\right) \wedge \bigwedge_{t=1}^{n} \varphi_{g}\left(\widetilde{a}_{g, t}, \widetilde{b}_{g, t}\right)$. From Lemma 2.6 we conclude that $\varphi_{f, g} \in \operatorname{Con} \mathcal{L}$.

Next lemma shows that $\varphi_{f, g}$ can be described as the join of a finite number of principal congruences and we use this result to determine the complement of $\theta(a, b)$. To obtain this description it is useful to remember facts $R_{1}$ ) an $R_{2}$ ) mentioned in [4] and to take into account the following:

Remark: Let $\mathcal{L}=(L, f, g) \in \mathbf{D K}_{n, m},(k, l)$ be an $m$-pair and $r \in \mathbb{N}_{0}$. Let $h \in\{f, g\}$. Then, for $x \in L_{1, m}^{h}$,

$$
\left\{\begin{array}{lll}
f^{r}\left(h^{k}(x)\right)=h^{l}(x), & f^{r}\left(h^{l}(x)\right)=h^{k}(x) & \text { if } r \text { is odd } \\
f^{r}\left(h^{k}(x)\right)=h^{k}(x), & f^{r}\left(h^{l}(x)\right)=h^{l}(x) & \text { if } r \text { is even } \\
g^{r}\left(h^{k}(x)\right)=h^{l}(x), & g^{r}\left(h^{l}(x)\right)=h^{k}(x) & \text { if } r \text { is odd } \\
g^{r}\left(h^{k}(x)\right)=h^{k}(x), & g^{r}\left(h^{l}(x)\right)=h^{l}(x) & \text { if } r \text { is even. }
\end{array}\right.
$$

Lemma 2.8. Let $\mathcal{L}=(L, f, g) \in \mathbf{D K}_{n, m}$ and $a, b \in L$ be such that $a \leq b$. Let $\widetilde{b}_{f, 0}=\widetilde{b}_{g, 0}=0$ and $\widetilde{a}_{f, n+1}=\widetilde{a}_{g, n+1}=1$ and $(k, l)$ be an m-pair.

Then,

$$
\varphi_{f, g}=\bigvee_{i, p=1}^{n+1} \bigvee_{j=i-1}^{n} \bigvee_{q=p-1}^{n}\left[\theta_{f}\left(x_{i, j, p, q}, y_{i, j, p, q}\right) \vee \theta_{g}\left(w_{i, j, p, q}, z_{i, j, p, q}\right)\right]
$$

where

$$
\begin{aligned}
& x_{i, j, p, q}=f^{l}\left(\widetilde{a}_{i}\right) \vee f^{k}\left(\widetilde{b}_{j}\right) \vee g^{l}\left(\widetilde{a}_{p}\right) \vee g^{k}\left(\widetilde{b}_{q}\right), \\
& y_{i, j, p, q}=x_{i, j, p, q} \vee\left(f^{k}\left(\widetilde{a}_{j+1}\right) \wedge f^{l}\left(\widetilde{b}_{i-1}\right) \wedge g^{k}\left(\widetilde{a}_{q+1}\right) \wedge g^{l}\left(\widetilde{b}_{p-1}\right)\right), \\
& w_{i, j, p, q}=f^{l}\left(\widetilde{a}_{i}\right) \vee f^{k}\left(\widetilde{b}_{j}\right) \vee\left(g^{k}\left(\widetilde{a}_{p}\right) \wedge g^{l}\left(\widetilde{b}_{q}\right) \wedge\left[g^{l}\left(\widetilde{a}_{q+1}\right) \vee g^{k}\left(\widetilde{b}_{p-1}\right)\right]\right), \\
& z_{i, j, p, q}=w_{i, j, p, q} \vee\left(f^{k}\left(\widetilde{a}_{j+1}\right) \wedge f^{l}\left(\widetilde{b}_{i-1}\right) \wedge g^{k}\left(\widetilde{a}_{p}\right) \wedge g^{l}\left(\widetilde{b}_{q}\right)\right) .
\end{aligned}
$$

Proof. We have $\varphi_{f, g}=\bigwedge_{s=1}^{n} \varphi_{f}\left(\widetilde{a}_{f, s}, \widetilde{b}_{f, s}\right) \wedge \bigwedge_{t=1}^{n} \varphi_{g}\left(\widetilde{a}_{g, t}, \widetilde{b}_{g, t}\right)$ and from [4] we know that

$$
\bigwedge_{s=1}^{n} \varphi_{f}\left(\widetilde{a}_{f, s}, \widetilde{b}_{f, s}\right)=\bigvee_{i=1}^{n+1} \bigvee_{j=i-1}^{n} \theta_{f}\left(f^{l}\left(\widetilde{a}_{i}\right) \vee f^{k}\left(\widetilde{b}_{j}\right), f^{l}\left(\widetilde{a}_{i}\right) \vee f^{k}\left(\widetilde{b}_{j}\right) \vee\left[f^{k}\left(\widetilde{a}_{j+1}\right) \wedge f^{l}\left(\widetilde{b}_{i-1}\right)\right]\right)
$$


and

$\bigwedge_{t=1}^{n} \varphi_{g}\left(\widetilde{a}_{g, t}, \widetilde{b}_{g, t}\right)=\bigvee_{p=1}^{n+1} \bigvee_{q=p-1}^{n} \theta_{g}\left(g^{l}\left(\widetilde{a}_{p}\right) \vee g^{k}\left(\widetilde{b}_{q}\right), g^{l}\left(\widetilde{a}_{p}\right) \vee g^{k}\left(\widetilde{b}_{q}\right) \vee\left[g^{k}\left(\widetilde{a}_{q+1}\right) \wedge g^{l}\left(\widetilde{b}_{p-1}\right)\right]\right)$.

By Lemma 1.3 and the remark we made before, it follows that:

$$
\begin{array}{r}
\bigwedge_{s=1}^{n} \varphi_{f}\left(\widetilde{a}_{f, s}, \widetilde{b}_{f, s}\right)=\bigvee_{i=1}^{n+1} \bigvee_{j=i-1}^{n}[ \\
\qquad \theta_{\text {lat }}\left(f^{l}\left(\widetilde{a}_{i}\right) \vee f^{k}\left(\widetilde{b}_{j}\right), f^{l}\left(\widetilde{a}_{i}\right) \vee f^{k}\left(\widetilde{b}_{j}\right) \vee\left[f^{k}\left(\widetilde{a}_{j+1}\right) \wedge f^{l}\left(\widetilde{b}_{i-1}\right)\right]\right) \\
\left.\vee \theta_{\text {lat }}\left(f^{k}\left(\widetilde{a}_{i}\right) \wedge f^{l}\left(\widetilde{b}_{j}\right) \wedge\left[f^{l}\left(\widetilde{a}_{j+1}\right) \vee f^{k}\left(\widetilde{b}_{i-1}\right)\right], f^{k}\left(\widetilde{a}_{i}\right) \wedge f^{l}\left(\widetilde{b}_{j}\right)\right)\right], \\
\bigwedge_{t=1}^{n} \varphi_{g}\left(\widetilde{a}_{g, t}, \widetilde{b}_{g, t}\right)=\bigvee_{p=1 q=p-1}^{n+1} \bigvee^{n}\left[\theta_{\text {lat }}\left(g^{l}\left(\widetilde{a}_{p}\right) \vee g^{k}\left(\widetilde{b}_{q}\right), g^{l}\left(\widetilde{a}_{p}\right) \vee g^{k}\left(\widetilde{b}_{q}\right) \vee\left[g^{k}\left(\widetilde{a}_{q+1}\right) \wedge g^{l}\left(\widetilde{b}_{p-1}\right)\right]\right)\right. \\
\left.\vee \theta_{\text {lat }}\left(g^{k}\left(\widetilde{a}_{p}\right) \wedge g^{l}\left(\widetilde{b}_{q}\right) \wedge\left[g^{l}\left(\widetilde{a}_{q+1}\right) \vee g^{k}\left(\widetilde{b}_{p-1}\right)\right], g^{k}\left(\widetilde{a}_{p}\right) \wedge g^{l}\left(\widetilde{b}_{q}\right)\right)\right] .
\end{array}
$$

Using $\left[4, R_{1}\right.$ ) and $R_{2}$ )] it is routine to prove the following identity (but we omit the proof since it is very long):

$$
\varphi_{f, g}=\bigvee_{i, p=1}^{n+1} \bigvee_{j=i-1}^{n} \bigvee_{q=p-1}^{n}\left(A_{i, j, p, q} \vee B_{i, j, p, q} \vee C_{i, j, p, q} \vee D_{i, j, p, q}\right),
$$

with

$A_{i, j, p, q}=\theta_{\text {lat }}\left(f^{l}\left(\widetilde{a}_{i}\right) \vee f^{k}\left(\widetilde{b}_{j}\right) \vee\left[g^{k}\left(\widetilde{a}_{p}\right) \wedge g^{l}\left(\widetilde{b}_{q}\right) \wedge\left(g^{k}\left(\widetilde{b}_{p-1}\right) \vee g^{l}\left(\widetilde{a}_{q+1}\right)\right)\right]\right.$, $\left.f^{l}\left(\widetilde{a}_{i}\right) \vee f^{k}\left(\widetilde{b}_{j}\right) \vee\left[g^{k}\left(\widetilde{a}_{p}\right) \wedge g^{l}\left(\widetilde{b}_{q}\right) \wedge\left(g^{k}\left(\widetilde{b}_{p-1}\right) \vee g^{l}\left(\widetilde{a}_{q+1}\right)\right)\right] \vee\left[f^{l}\left(\widetilde{b}_{i-1}\right) \wedge f^{k}\left(\widetilde{a}_{j+1}\right) \wedge g^{k}\left(\widetilde{a}_{p}\right) \wedge g^{l}\left(\widetilde{b}_{q}\right)\right]\right) ;$

$B_{i, j, p, q}=\theta_{\text {lat }}\left(f^{k}\left(\widetilde{a}_{i}\right) \wedge f^{l}\left(\widetilde{b}_{j}\right) \wedge\left[g^{l}\left(\widetilde{a}_{p}\right) \vee g^{k}\left(\widetilde{b}_{q}\right) \vee\left(g^{l}\left(\widetilde{b}_{p-1}\right) \wedge g^{k}\left(\widetilde{a}_{q+1}\right)\right)\right]\right.$,

$\left.f^{k}\left(\widetilde{a}_{i}\right) \wedge f^{l}\left(\widetilde{b}_{j}\right) \wedge\left[g^{l}\left(\widetilde{a}_{p}\right) \vee g^{k}\left(\widetilde{b}_{q}\right) \vee\left(g^{l}\left(\widetilde{b}_{p-1}\right) \wedge g^{k}\left(\widetilde{a}_{q+1}\right)\right)\right] \wedge\left[f^{k}\left(\widetilde{b}_{i-1}\right) \vee f^{l}\left(\widetilde{a}_{j+1}\right) \vee g^{l}\left(\widetilde{a}_{p}\right) \vee g^{k}\left(\widetilde{b}_{q}\right)\right]\right) ;$

$C_{i, j, p, q}=\theta_{\text {lat }}\left(f^{l}\left(\widetilde{a}_{i}\right) \vee f^{k}\left(\widetilde{b}_{j}\right) \vee g^{l}\left(\widetilde{a}_{p}\right) \vee g^{k}\left(\widetilde{b}_{q}\right)\right.$,

$\left.f^{l}\left(\widetilde{a}_{i}\right) \vee f^{k}\left(\widetilde{b}_{j}\right) \vee g^{l}\left(\widetilde{a}_{p}\right) \vee g^{k}\left(\widetilde{b}_{q}\right) \vee\left[f^{l}\left(\widetilde{b}_{i-1}\right) \wedge f^{k}\left(\widetilde{a}_{j+1}\right) \wedge g^{l}\left(\widetilde{b}_{p-1}\right) \wedge g^{k}\left(\widetilde{a}_{q+1}\right)\right]\right) ;$

$D_{i, j, p, q}=\theta_{\text {lat }}\left(f^{k}\left(\widetilde{a}_{i}\right) \wedge f^{l}\left(\widetilde{b}_{j}\right) \wedge g^{k}\left(\widetilde{a}_{p}\right) \wedge g^{l}\left(\widetilde{b}_{q}\right) \wedge\left[f^{k}\left(\widetilde{b}_{i-1}\right) \vee f^{l}\left(\widetilde{a}_{j+1}\right) \vee g^{k}\left(\widetilde{b}_{p-1}\right) \vee g^{l}\left(\widetilde{a}_{q+1}\right)\right]\right.$, $\left.f^{k}\left(\widetilde{a}_{i}\right) \wedge f^{l}\left(\widetilde{b}_{j}\right) \wedge g^{k}\left(\widetilde{a}_{p}\right) \wedge g^{l}\left(\widetilde{b}_{q}\right)\right)$.

Now, from Lemma 1.3 it follows that

$$
\varphi_{f, g}=\bigvee_{i, p=1}^{n+1} \bigvee_{j=i-1}^{n} \bigvee_{q=p-1}^{n}\left(\theta_{f}\left(x_{i, j, p, q}, y_{i, j, p, q}\right) \vee \theta_{g}\left(w_{i, j, p, q}, z_{i, j, p, q}\right)\right),
$$

where

$$
\begin{aligned}
& x_{i, j, p, q}=f^{l}\left(\widetilde{a}_{i}\right) \vee f^{k}\left(\widetilde{b}_{j}\right) \vee g^{l}\left(\widetilde{a}_{p}\right) \vee g^{k}\left(\widetilde{b}_{q}\right), \\
& y_{i, j, p, q}=x_{i, j, p, q} \vee\left(f^{k}\left(\widetilde{a}_{j+1}\right) \wedge f^{l}\left(\widetilde{b}_{i-1}\right) \wedge g^{k}\left(\widetilde{a}_{q+1}\right) \wedge g^{l}\left(\widetilde{b}_{p-1}\right)\right), \\
& w_{i, j, p, q}=f^{l}\left(\widetilde{a}_{i}\right) \vee f^{k}\left(\widetilde{b}_{j}\right) \vee\left(g^{k}\left(\widetilde{a}_{p}\right) \wedge g^{l}\left(\widetilde{b}_{q}\right) \wedge\left[g^{l}\left(\widetilde{a}_{q+1}\right) \vee g^{k}\left(\widetilde{b}_{p-1}\right)\right]\right), \\
& z_{i, j, p, q}=w_{i, j, p, q} \vee\left(f^{k}\left(\widetilde{a}_{j+1}\right) \wedge f^{l}\left(\widetilde{b}_{i-1}\right) \wedge g^{k}\left(\widetilde{a}_{p}\right) \wedge g^{l}\left(\widetilde{b}_{q}\right)\right) .
\end{aligned}
$$


Theorem 2.9. Let $\mathcal{L}=(L, f, g) \in \mathbf{D K}_{n, m}$ and $a, b \in L$ be such that $a \leq b$. Let $(k, l)$ be an m-pair.

Then,

(a) $\theta(a, b) \vee \varphi_{f, g}=\mathbf{1}$

(b) if $\theta(a, b)$ is complemented, then necessarily $\theta(a, b)^{\prime}=\varphi_{f, g}$.

Proof. (a) By Lemma 1.8, we have $\theta(a, b)=\bigvee_{s=1}^{n} \theta_{f}\left(\widetilde{a}_{f, s}, \widetilde{b}_{f, s}\right) \vee \bigvee_{t=1}^{n} \theta_{g}\left(\widetilde{a}_{g, t}, \widetilde{b}_{g, t}\right)$ and from [4, Theorem 2.7] we know that, for all $s, t \in\{1, \ldots, n\}$, $\theta_{f}\left(\widetilde{a}_{f, s}, \widetilde{b}_{f, s}\right) \vee \varphi_{f}\left(\widetilde{a}_{f, s,} \widetilde{b}_{f, s}\right)=\mathbf{1}$ and $\theta_{g}\left(\widetilde{a}_{g, t}, \widetilde{b}_{g, t}\right) \vee \varphi_{g}\left(\widetilde{a}_{g, t}, \widetilde{b}_{g, t}\right)=\mathbf{1}$.

Consequently,

$$
\begin{aligned}
\theta \vee \varphi_{f, g}= & {\left[\bigvee_{s=1}^{n} \theta_{f}\left(\widetilde{a}_{f, s}, \widetilde{b}_{f, s}\right) \vee \bigvee_{t=1}^{n} \theta_{g}\left(\widetilde{a}_{g, t}, \widetilde{b}_{g, t}\right)\right] \vee\left[\bigwedge_{u=1}^{n} \varphi_{f}\left(\widetilde{a}_{f, u}, \widetilde{b}_{f, u}\right) \wedge \bigwedge_{v=1}^{n} \varphi_{g}\left(\widetilde{a}_{g, v}, \widetilde{b}_{g, v}\right)\right] } \\
= & \bigwedge_{u=1}^{n}\left(\varphi_{f}\left(\widetilde{a}_{f, u}, \widetilde{b}_{g, u}\right) \vee \theta_{f}\left(\widetilde{a}_{f, u}, \widetilde{b}_{f, u}\right) \vee \bigvee_{s=1, s \neq u}^{n} \theta_{f}\left(\widetilde{a}_{f, s} \widetilde{b}_{f, s}\right) \vee \bigvee_{t=1}^{n} \theta_{g}\left(\widetilde{a}_{g, t} \widetilde{b}_{g, t}\right)\right) \\
& \wedge \bigwedge_{v=1}^{n}\left(\varphi_{g}\left(\widetilde{a}_{g, v}, \widetilde{b}_{g, v}\right) \vee \theta_{g}\left(\widetilde{a}_{g, v}, \widetilde{b}_{g, v}\right) \vee \bigvee_{s=1}^{n} \theta_{f}\left(\widetilde{a}_{f, s}, \widetilde{b}_{f, s}\right) \vee \bigvee_{t=1, t \neq v}^{n} \theta_{g}\left(\widetilde{a}_{g, t}, \widetilde{b}_{g, t}\right)\right) \\
= & \mathbf{1 .}
\end{aligned}
$$

(b) Suppose now that $\theta(a, b)$ is complemented. From (a) it follows that $\theta(a, b)^{\prime} \leq \varphi_{f, g}$. It remains to prove that $\varphi_{f, g} \leq \theta(a, b)^{\prime}$.

As we have already seen $\left(\left.\theta(a, b)\right|_{f^{m}(L)}\right)^{\prime}=\left.\varphi_{f, g}\right|_{f^{m}(L)}$.

Let $\widetilde{b}_{f, 0}=\widetilde{b}_{g, 0}=0$ and $\widetilde{a}_{f, n+1}=\widetilde{a}_{g, n+1}=1$. By Lemma 2.8 we have

$$
\varphi_{f, g}=\bigvee_{i, p=1}^{n+1} \bigvee_{j=i-1}^{n} \bigvee_{q=p-1}^{n}\left[\theta_{f}\left(x_{i, j, p, q}, y_{i, j, p, q}\right) \vee \theta_{g}\left(w_{i, j, p, q}, z_{i, j, p, q}\right)\right],
$$

where

$$
\begin{aligned}
& x_{i, j, p, q}=f^{l}\left(\widetilde{a}_{i}\right) \vee f^{k}\left(\widetilde{b}_{j}\right) \vee g^{l}\left(\widetilde{a}_{p}\right) \vee g^{k}\left(\widetilde{b}_{q}\right), \\
& y_{i, j, p, q}=x_{i, j, p, q} \vee\left(f^{k}\left(\widetilde{a}_{j+1}\right) \wedge f^{l}\left(\widetilde{b}_{i-1}\right) \wedge g^{k}\left(\widetilde{a}_{q+1}\right) \wedge g^{l}\left(\widetilde{b}_{p-1}\right)\right), \\
& w_{i, j, p, q}=f^{l}\left(\widetilde{a}_{i}\right) \vee f^{k}\left(\widetilde{b}_{j}\right) \vee\left(g^{k}\left(\widetilde{a}_{p}\right) \wedge g^{l}\left(\widetilde{b}_{q}\right) \wedge\left[g^{l}\left(\widetilde{a}_{q+1}\right) \vee g^{k}\left(\widetilde{b}_{p-1}\right)\right]\right), \\
& z_{i, j, p, q}=w_{i, j, p, q} \vee\left(f^{k}\left(\widetilde{a}_{j+1}\right) \wedge f^{l}\left(\widetilde{b}_{i-1}\right) \wedge g^{k}\left(\widetilde{a}_{p}\right) \wedge g^{l}\left(\widetilde{b}_{q}\right)\right) .
\end{aligned}
$$

From Lemma 2.6 we know that $\theta_{f}\left(x_{i, j, p, q}, y_{i, j, p, q}\right)$ and $\theta_{g}\left(w_{i, j, p, q}, z_{i, j, p, q}\right)$ are elements of $\operatorname{Con} \mathcal{L}$. So $\varphi_{f, g}$ is the least congruence of $\mathcal{L}$ that identifies each pair $\left(x_{i, j, p, q}, y_{i, j, p, q}\right)$ and each pair $\left(w_{i, j, p, q}, z_{i, j, p, q}\right)$.

Taking into account Lemma 2.5 we have $\left(\left.\theta(a, b)\right|_{f^{m}(L)}\right)^{\prime}=\left.\theta(a, b)^{\prime}\right|_{f^{m}(L)}$. So $\left.\varphi_{f, g}\right|_{f^{m}(L)}=\left.\theta(a, b)^{\prime}\right|_{f^{m}(L)}$ and, consequently, $\theta(a, b)^{\prime}$ also identifies each of those pairs. Therefore $\varphi_{f, g} \leq \theta(a, b)^{\prime}$ and we may conclude that $\theta(a, b)^{\prime}=\varphi_{f, g}$. 
A double Ockham algebra $\mathcal{L}=(L, f, g)$ that satisfies id $\leq f^{2}, g^{2} \leq \mathrm{id}, f g=g^{2}$ and $g f=f^{2}$ is called a double MS-algebra. Since every double MS-algebra is a double $K_{1,1}$-algebra, we can establish Theorem 14.5 of [3] as a corollary of the previous theorem. Thus we have:

Corollary 2.10. Let $\mathcal{L}=(L, f, g)$ be a double MS-algebra and let $a, b \in L$ be such that $a \leq b$.

Let

$$
\begin{aligned}
\varphi_{f, g}=\left[\theta_{f}\left(f^{2}(b) \vee f(a), 1\right) \vee \theta_{f}\left(f^{2}(b), f^{2}(b) \vee f(b)\right) \vee\right. & \left.\theta_{f}\left(f(a), f(a) \vee f^{2}(a)\right)\right] \\
& \wedge\left[\theta_{g}\left(g^{2}(b) \vee g(a), 1\right) \vee \theta_{g}\left(g^{2}(b), g^{2}(b) \vee g(b)\right) \vee \theta_{g}\left(g(a), g(a) \vee g^{2}(a)\right)\right] .
\end{aligned}
$$

Then

(a) $\theta(a, b) \vee \varphi_{f, g}=\mathbf{1}$

(b) if $\theta(a, b)$ is complemented, then $\theta(a, b)^{\prime}=\varphi_{f, g}$.

We finish this paper establishing a necessary and sufficient condition for a principal congruence defined on a double $\mathrm{K}_{n, m}$-algebra to be complemented.

Theorem 2.11. Let $\mathcal{L}=(L, f) \in \mathbf{D K}_{n, m}$ and $a, b \in L$ be such that $a \leq b$. Let $(k, l)$ be an m-pair. Let $\widetilde{b}_{f, 0}=\widetilde{b}_{g, 0}=0$ and $\widetilde{a}_{f, n+1}=\widetilde{a}_{g, n+1}=1$. Then, $\theta(a, b)$ is complemented if and only if for all $s \in\{1, \ldots, n\}$, all $\left(x_{s}, y_{s}\right) \in\left\{\left(\widetilde{a}_{f, s}, \widetilde{b}_{f, s}\right),\left(\widetilde{a}_{g, s}, \widetilde{b}_{g, s}\right)\right\}$ and all $i, p \in\{1, \ldots, n+1\}$ we have:

$y_{s} \wedge f^{k}\left(\widetilde{a}_{j+1}\right) \wedge f^{l}\left(\widetilde{b}_{i-1}\right) \wedge g^{k}\left(\widetilde{a}_{q+1}\right) \wedge g^{l}\left(\widetilde{b}_{p-1}\right) \leq x_{s} \vee f^{l}\left(\widetilde{a}_{i}\right) \vee f^{k}\left(\widetilde{b}_{j}\right) \vee g^{l}\left(\widetilde{a}_{p}\right) \vee g^{k}\left(\widetilde{b}_{q}\right)$, for all $j \in\{i-1, \ldots, n\}$ and $q \in\{p-1, \ldots, n\}$,

$y_{s} \wedge f^{k}\left(\widetilde{a}_{j+1}\right) \wedge f^{l}\left(\widetilde{b}_{i-1}\right) \wedge g^{k}\left(\widetilde{a}_{p}\right) \wedge g^{l}\left(\widetilde{b}_{q}\right) \leq x_{s} \vee f^{l}\left(\widetilde{a}_{i}\right) \vee f^{k}\left(\widetilde{b}_{j}\right) \vee g^{l}\left(\widetilde{a}_{q+1}\right) \vee g^{k}\left(\widetilde{b}_{p-1}\right)$, for all $j \in\{i-1, \ldots, n\}$ and $q \in\{p, \ldots, n\}$,

$y_{s} \wedge f^{k}\left(\widetilde{a}_{i}\right) \wedge f^{l}\left(\widetilde{b}_{j}\right) \wedge g^{k}\left(\widetilde{a}_{q+1}\right) \wedge g^{l}\left(\widetilde{b}_{p-1}\right) \leq x_{s} \vee f^{l}\left(\widetilde{a}_{j+1}\right) \vee f^{k}\left(\widetilde{b}_{i-1}\right) \vee g^{l}\left(\widetilde{a}_{p}\right) \vee g^{k}\left(\widetilde{b}_{q}\right)$, for all $j \in\{i, \ldots, n\}$ and $q \in\{p-1, \ldots, n\}$,

$y_{s} \wedge f^{k}\left(\widetilde{a}_{i}\right) \wedge f^{l}\left(\widetilde{b}_{j}\right) \wedge g^{k}\left(\widetilde{a}_{p}\right) \wedge g^{l}\left(\widetilde{b}_{q}\right) \leq x_{s} \vee f^{l}\left(\widetilde{a}_{j+1}\right) \vee f^{k}\left(\widetilde{b}_{i-1}\right) \vee g^{l}\left(\widetilde{a}_{q+1}\right) \vee g^{k}\left(\widetilde{b}_{p-1}\right)$, for all $j \in\{i, \ldots, n\}$ and $q \in\{p, \ldots, n\}$.

Proof. By Lemma 1.8 we have $\theta(a, b)=\bigvee_{s=1}^{n} \theta_{f}\left(\widetilde{a}_{f, s}, \widetilde{b}_{f, s}\right) \vee \bigvee_{t=1}^{n} \theta_{g}\left(\widetilde{a}_{g, t}, \widetilde{b}_{g, t}\right)$ and, from Theorem 2.9 it follows that $\theta(a, b)$ is complemented if and only if $\theta(a, b) \wedge \varphi_{f, g}=\mathbf{0}$. By Lemma 2.8 we know that

$$
\begin{aligned}
\varphi_{f, g}= & \left(\bigvee_{i=1}^{n+1} \bigvee_{j=i-1}^{n} \theta_{f}\left(f^{l}\left(\widetilde{a}_{i}\right) \vee f^{k}\left(\widetilde{b}_{j}\right), f^{l}\left(\widetilde{a}_{i}\right) \vee f^{k}\left(\widetilde{b}_{j}\right) \vee\left[f^{k}\left(\widetilde{a}_{j+1}\right) \wedge f^{l}\left(\widetilde{b}_{i-1}\right)\right]\right)\right) \\
& \wedge\left(\bigvee_{p=1}^{n+1} \bigvee_{q=p-1}^{n} \theta_{g}\left(g^{l}\left(\widetilde{a}_{p}\right) \vee g^{k}\left(\widetilde{b}_{q}\right), g^{l}\left(\widetilde{a}_{p}\right) \vee g^{k}\left(\widetilde{b}_{q}\right) \vee\left[g^{k}\left(\widetilde{a}_{q+1}\right) \wedge g^{l}\left(\widetilde{b}_{p-1}\right)\right]\right)\right)
\end{aligned}
$$

with $\widetilde{b}_{f, 0}=\widetilde{b}_{g, 0}=0$ and $\widetilde{a}_{f, n+1}=\widetilde{a}_{g, n+1}=1$. 
Then $\theta(a, b)$ is complemented if and only if, for all $s, t \in\{1, \ldots, n\}$, $i, p \in\{1, \ldots, n+1\}, j \in\{i-1, \ldots, n\}$ and $q \in\{p-1, \ldots, n\}$,

$$
\begin{aligned}
\theta_{f}\left(\widetilde{a}_{f, s}, \widetilde{b}_{f, s}\right) & \wedge \theta_{f}\left(f^{l}\left(\widetilde{a}_{i}\right) \vee f^{k}\left(\widetilde{b}_{j}\right), f^{l}\left(\widetilde{a}_{i}\right) \vee f^{k}\left(\widetilde{b}_{j}\right) \vee\left[f^{k}\left(\widetilde{a}_{j+1}\right) \wedge f^{l}\left(\widetilde{b}_{i-1}\right)\right]\right) \\
& \wedge \theta_{g}\left(g^{l}\left(\widetilde{a}_{p}\right) \vee g^{k}\left(\widetilde{b}_{q}\right), g^{l}\left(\widetilde{a}_{p}\right) \vee g^{k}\left(\widetilde{b}_{q}\right) \vee\left[g^{k}\left(\widetilde{a}_{q+1}\right) \wedge g^{l}\left(\widetilde{b}_{p-1}\right)\right]\right)=\mathbf{0}
\end{aligned}
$$

and

$$
\begin{aligned}
\theta_{g}\left(\widetilde{a}_{g, t}, \widetilde{b}_{g, t}\right) & \wedge \theta_{f}\left(f^{l}\left(\widetilde{a}_{i}\right) \vee f^{k}\left(\widetilde{b}_{j}\right), f^{l}\left(\widetilde{a}_{i}\right) \vee f^{k}\left(\widetilde{b}_{j}\right) \vee\left[f^{k}\left(\widetilde{a}_{j+1}\right) \wedge f^{l}\left(\widetilde{b}_{i-1}\right)\right]\right) \\
& \wedge \theta_{g}\left(g^{l}\left(\widetilde{a}_{p}\right) \vee g^{k}\left(\widetilde{b}_{q}\right), g^{l}\left(\widetilde{a}_{p}\right) \vee g^{k}\left(\widetilde{b}_{q}\right) \vee\left[g^{k}\left(\widetilde{a}_{q+1}\right) \wedge g^{l}\left(\widetilde{b}_{p-1}\right)\right]\right)=\mathbf{0} .
\end{aligned}
$$

By Lemma 1.3 and since $\widetilde{a}_{f, s}, \widetilde{b}_{f, s} \in L_{1, m}^{f}$ and $\widetilde{a}_{g, t}, \widetilde{b}_{g, t} \in L_{1, m}^{g}$, it follows that, for all $s \in\{1, \ldots, n\}, i, p \in\{1, \ldots, n+1\}, j \in\{i-1, \ldots, n\}$ and $q \in\{p-1, \ldots, n\}$,

$$
\begin{aligned}
\theta_{f}\left(\widetilde{a}_{f, s}, \widetilde{b}_{f, s}\right) & \wedge \theta_{f}\left(f^{l}\left(\widetilde{a}_{i}\right) \vee f^{k}\left(\widetilde{b}_{j}\right), f^{l}\left(\widetilde{a}_{i}\right) \vee f^{k}\left(\widetilde{b}_{j}\right) \vee\left[f^{k}\left(\widetilde{a}_{j+1}\right) \wedge f^{l}\left(\widetilde{b}_{i-1}\right)\right]\right) \\
& \wedge \theta_{g}\left(g^{l}\left(\widetilde{a}_{p}\right) \vee g^{k}\left(\widetilde{b}_{q}\right), g^{l}\left(\widetilde{a}_{p}\right) \vee g^{k}\left(\widetilde{b}_{q}\right) \vee\left[g^{k}\left(\widetilde{a}_{q+1}\right) \wedge g^{l}\left(\widetilde{b}_{p-1}\right)\right]\right)=\mathbf{0}
\end{aligned}
$$

if and only if

$$
\begin{aligned}
& {\left[\bigvee_{r=0}^{m+1} \theta_{\text {lat }}\left(f^{r}\left(\widetilde{a}_{s}\right), f^{r}\left(\widetilde{b}_{s}\right)\right)\right]} \\
& \wedge\left[\theta_{\text {lat }}\left(f^{l}\left(\widetilde{a}_{i}\right) \vee f^{k}\left(\widetilde{b}_{j}\right), f^{l}\left(\widetilde{a}_{i}\right) \vee f^{k}\left(\widetilde{b}_{j}\right) \vee\left[f^{k}\left(\widetilde{a}_{j+1}\right) \wedge f^{l}\left(\widetilde{b}_{i-1}\right)\right]\right)\right. \\
& \left.\quad \vee \theta_{\text {lat }}\left(f^{k}\left(\widetilde{a}_{i}\right) \wedge f^{l}\left(\widetilde{b}_{j}\right) \wedge\left[f^{l}\left(\widetilde{a}_{j+1}\right) \vee f^{k}\left(\widetilde{b}_{i-1}\right)\right], f^{k}\left(\widetilde{a}_{i}\right) \wedge f^{l}\left(\widetilde{b}_{j}\right)\right)\right] \\
& \wedge\left[\theta_{\text {lat }}\left(g^{l}\left(\widetilde{a}_{p}\right) \vee g^{k}\left(\widetilde{b}_{q}\right), g^{l}\left(\widetilde{a}_{p}\right) \vee g^{k}\left(\widetilde{b}_{q}\right) \vee\left[g^{k}\left(\widetilde{a}_{q+1}\right) \wedge g^{l}\left(\widetilde{b}_{p-1}\right)\right]\right)\right. \\
& \left.\quad \vee \theta_{\text {lat }}\left(g^{k}\left(\widetilde{a}_{p}\right) \wedge g^{l}\left(\widetilde{b}_{q}\right) \wedge\left[g^{l}\left(\widetilde{a}_{q+1}\right) \vee g^{k}\left(\widetilde{b}_{p-1}\right)\right], g^{k}\left(\widetilde{a}_{p}\right) \wedge g^{l}\left(\widetilde{b}_{q}\right)\right)\right]=\mathbf{0 .} .
\end{aligned}
$$

Now, using $\left[4, \mathrm{R}_{1}\right.$ ) and $\left.\left.\mathrm{R}_{2}\right)\right]$ it is easy we conclude that the previous identity follows if and only if, for all $r \in\{0, \ldots, m+1\}$,

a) $\quad f^{r}\left(\widetilde{b}_{s}\right) \wedge f^{k}\left(\widetilde{a}_{j+1}\right) \wedge f^{l}\left(\widetilde{b}_{i-1}\right) \wedge g^{k}\left(\widetilde{a}_{q+1}\right) \wedge g^{l}\left(\widetilde{b}_{p-1}\right)$

$$
\leq f^{r}\left(\widetilde{a}_{s}\right) \vee f^{l}\left(\widetilde{a}_{i}\right) \vee f^{k}\left(\widetilde{b}_{j}\right) \vee g^{l}\left(\widetilde{a}_{p}\right) \vee g^{k}\left(\widetilde{b}_{q}\right),
$$

b) $\quad f^{r}\left(\widetilde{b}_{s}\right) \wedge f^{k}\left(\widetilde{a}_{j+1}\right) \wedge f^{l}\left(\widetilde{b}_{i-1}\right) \wedge g^{k}\left(\widetilde{a}_{p}\right) \wedge g^{l}\left(\widetilde{b}_{q}\right)$

$$
\leq f^{r}\left(\widetilde{a}_{s}\right) \vee f^{l}\left(\widetilde{a}_{i}\right) \vee f^{k}\left(\widetilde{b}_{j}\right) \vee g^{l}\left(\widetilde{a}_{q+1}\right) \vee g^{k}\left(\widetilde{b}_{p-1}\right),
$$

c)

$$
\begin{aligned}
& f^{r}\left(\widetilde{b}_{s}\right) \wedge f^{k}\left(\widetilde{a}_{i}\right) \wedge f^{l}\left(\widetilde{b}_{j}\right) \wedge g^{k}\left(\widetilde{a}_{q+1}\right) \wedge g^{l}\left(\widetilde{b}_{p-1}\right) \\
\leq & f^{r}\left(\widetilde{a}_{s}\right) \vee f^{l}\left(\widetilde{a}_{j+1}\right) \vee f^{k}\left(\widetilde{b}_{i-1}\right) \vee g^{l}\left(\widetilde{a}_{p}\right) \vee g^{k}\left(\widetilde{b}_{q}\right),
\end{aligned}
$$

and

d) $\quad f^{r}\left(\widetilde{b}_{s}\right) \wedge f^{k}\left(\widetilde{a}_{i}\right) \wedge f^{l}\left(\widetilde{b}_{j}\right) \wedge g^{k}\left(\widetilde{a}_{p}\right) \wedge g^{l}\left(\widetilde{b}_{q}\right)$

$$
\leq f^{r}\left(\widetilde{a}_{s}\right) \vee f^{l}\left(\widetilde{a}_{j+1}\right) \vee f^{k}\left(\widetilde{b}_{i-1}\right) \vee g^{l}\left(\widetilde{a}_{q+1}\right) \vee g^{k}\left(\widetilde{b}_{p-1}\right) \text {. }
$$


These inequalities are trivial when $r$ is odd. If $r$ is even, we have already seen that, $f^{r}\left(f^{k}(x)\right)=f^{k}(x), f^{r}\left(f^{l}(x)\right)=f^{l}(x), f^{r}\left(g^{k}(y)\right)=g^{k}(y)$ and $f^{r}\left(g^{l}(y)\right)=g^{l}(y)$, for all $x \in L_{1, m}^{f}$ and $y \in L_{1, m}^{g}$. So, conditions a), b), c) and d) are equivalent, respectively, to 1), 2), 3) and 4) below:

1)

$$
\begin{aligned}
& \widetilde{b}_{f, s} \wedge f^{k}\left(\widetilde{a}_{j+1}\right) \wedge f^{l}\left(\widetilde{b}_{i-1}\right) \wedge g^{k}\left(\widetilde{a}_{q+1}\right) \wedge g^{l}\left(\widetilde{b}_{p-1}\right) \\
\leq & \widetilde{a}_{f, s} \vee f^{l}\left(\widetilde{a}_{i}\right) \vee f^{k}\left(\widetilde{b}_{j}\right) \vee g^{l}\left(\widetilde{a}_{p}\right) \vee g^{k}\left(\widetilde{b}_{q}\right),
\end{aligned}
$$

$$
\begin{aligned}
& \widetilde{b}_{f, s} \wedge f^{k}\left(\widetilde{a}_{j+1}\right) \wedge f^{l}\left(\widetilde{b}_{i-1}\right) \wedge g^{k}\left(\widetilde{a}_{p}\right) \wedge g^{l}\left(\widetilde{b}_{q}\right) \\
\leq & \widetilde{a}_{f, s} \vee f^{l}\left(\widetilde{a}_{i}\right) \vee f^{k}\left(\widetilde{b}_{j}\right) \vee g^{l}\left(\widetilde{a}_{q+1}\right) \vee g^{k}\left(\widetilde{b}_{p-1}\right),
\end{aligned}
$$

$$
\begin{aligned}
& \widetilde{b}_{f, s} \wedge f^{k}\left(\widetilde{a}_{i}\right) \wedge f^{l}\left(\widetilde{b}_{j}\right) \wedge g^{k}\left(\widetilde{a}_{q+1}\right) \wedge g^{l}\left(\widetilde{b}_{p-1}\right) \\
\leq & \widetilde{a}_{f, s} \vee f^{l}\left(\widetilde{a}_{j+1}\right) \vee f^{k}\left(\widetilde{b}_{i-1}\right) \vee g^{l}\left(\widetilde{a}_{p}\right) \vee g^{k}\left(\widetilde{b}_{q}\right),
\end{aligned}
$$

4)

$$
\begin{aligned}
& \widetilde{b}_{f, s} \wedge f^{k}\left(\widetilde{a}_{i}\right) \wedge f^{l}\left(\widetilde{b}_{j}\right) \wedge g^{k}\left(\widetilde{a}_{p}\right) \wedge g^{l}\left(\widetilde{b}_{q}\right) \\
\leq & \widetilde{a}_{f, s} \vee f^{l}\left(\widetilde{a}_{j+1}\right) \vee f^{k}\left(\widetilde{b}_{i-1}\right) \vee g^{l}\left(\widetilde{a}_{q+1}\right) \vee g^{k}\left(\widetilde{b}_{p-1}\right) .
\end{aligned}
$$

Conditions 1) and 2) are equal when $q=p-1$ (the same happens with 3) and 4)). For $j=i-1$ we also have that 1 ) coincide with 3 ) and 2) coincide with 4)).

Given $t \in\{1, \ldots, n\}, i, p \in\{1, \ldots, n+1\}, j \in\{i-1, \ldots, n\}$ and $q \in\{p-1, \ldots, n\}$, we have

$$
\begin{aligned}
\theta_{g}\left(\widetilde{a}_{g, t}, \widetilde{b}_{g, t}\right) & \wedge \theta_{f}\left(f^{l}\left(\widetilde{a}_{i}\right) \vee f^{k}\left(\widetilde{b}_{j}\right), f^{l}\left(\widetilde{a}_{i}\right) \vee f^{k}\left(\widetilde{b}_{j}\right) \vee\left[f^{k}\left(\widetilde{a}_{j+1}\right) \wedge f^{l}\left(\widetilde{b}_{i-1}\right)\right]\right) \\
& \wedge \theta_{g}\left(g^{l}\left(\widetilde{a}_{p}\right) \vee g^{k}\left(\widetilde{b}_{q}\right), g^{l}\left(\widetilde{a}_{p}\right) \vee g^{k}\left(\widetilde{b}_{q}\right) \vee\left[g^{k}\left(\widetilde{a}_{q+1}\right) \wedge g^{l}\left(\widetilde{b}_{p-1}\right)\right]\right)=\mathbf{0} .
\end{aligned}
$$

if and only if are satisfied conditions analogous to 1), 2), 3) and 4).

Then $\theta(a, b)$ is complemented if and only if for all $s \in\{1, \ldots, n\}$, all $\left(x_{s}, y_{s}\right) \in\left\{\left(\widetilde{a}_{f, s}, \widetilde{b}_{f, s}\right),\left(\widetilde{a}_{g, s}, \widetilde{b}_{g, s}\right)\right\}$ and all $i, p \in\{1, \ldots, n+1\}$ the following conditions hold:

$y_{s} \wedge f^{k}\left(\widetilde{a}_{j+1}\right) \wedge f^{l}\left(\widetilde{b}_{i-1}\right) \wedge g^{k}\left(\widetilde{a}_{q+1}\right) \wedge g^{l}\left(\widetilde{b}_{p-1}\right) \leq x_{s} \vee f^{l}\left(\widetilde{a}_{i}\right) \vee f^{k}\left(\widetilde{b}_{j}\right) \vee g^{l}\left(\widetilde{a}_{p}\right) \vee g^{k}\left(\widetilde{b}_{q}\right)$, for all $j \in\{i-1, \ldots, n\}$ and $q \in\{p-1, \ldots, n\}$,

$y_{s} \wedge f^{k}\left(\widetilde{a}_{j+1}\right) \wedge f^{l}\left(\widetilde{b}_{i-1}\right) \wedge g^{k}\left(\widetilde{a}_{p}\right) \wedge g^{l}\left(\widetilde{b}_{q}\right) \leq x_{s} \vee f^{l}\left(\widetilde{a}_{i}\right) \vee f^{k}\left(\widetilde{b}_{j}\right) \vee g^{l}\left(\widetilde{a}_{q+1}\right) \vee g^{k}\left(\widetilde{b}_{p-1}\right)$, for all $j \in\{i-1, \ldots, n\}$ and $q \in\{p, \ldots, n\}$,

$y_{s} \wedge f^{k}\left(\widetilde{a}_{i}\right) \wedge f^{l}\left(\widetilde{b}_{j}\right) \wedge g^{k}\left(\widetilde{a}_{q+1}\right) \wedge g^{l}\left(\widetilde{b}_{p-1}\right) \leq x_{s} \vee f^{l}\left(\widetilde{a}_{j+1}\right) \vee f^{k}\left(\widetilde{b}_{i-1}\right) \vee g^{l}\left(\widetilde{a}_{p}\right) \vee g^{k}\left(\widetilde{b}_{q}\right)$, for all $j \in\{i, \ldots, n\}$ and $q \in\{p-1, \ldots, n\}$,

$y_{s} \wedge f^{k}\left(\widetilde{a}_{i}\right) \wedge f^{l}\left(\widetilde{b}_{j}\right) \wedge g^{k}\left(\widetilde{a}_{p}\right) \wedge g^{l}\left(\widetilde{b}_{q}\right) \leq x_{s} \vee f^{l}\left(\widetilde{a}_{j+1}\right) \vee f^{k}\left(\widetilde{b}_{i-1}\right) \vee g^{l}\left(\widetilde{a}_{q+1}\right) \vee g^{k}\left(\widetilde{b}_{p-1}\right)$, for all $j \in\{i, \ldots, n\}$ and $q \in\{p, \ldots, n\}$. 


\section{Acknowledgements}

The support from the Portuguese Foundation for Science and Technology through the research program POCTI is gratefully acknowledged.

The author is pleased to acknowledge useful discussion with her supervisor, Dr. Margarida Sequeira, and also would like to thank Prof. M. Paula Marques Smith for the valuable suggestions on the writing of this paper.

\section{REFERENCES}

[1] Berman, J. - Distributive lattices with an additional unary operation, Aequat. Math. 16 (1977), 165-171.

[2] Blyth, T.S., A.S.A. Noor and Varlet, J.C. - Congruences on double MS-algebras, Bull. Soc. Roy. Sci. Liège 56 (1987), 143-152.

[3] Blyth, T.S. and Varlet, J.C. - Ockham algebras, Oxford Science Publications, 1995.

[4] Mendes, C. - Complemented congruences on Ockham algebras, accepted for publication in Algebra Universalis.

[5] Sequeira, M. - Double $\mathrm{MS}_{n}$-algebras and double $\mathrm{K}_{n, m}$-algebras, Glasgow Math. (1993), $189-201$.

Carla Mendes

Centro de Matemática, Universidade do Minho, 4710-057 Braga, Portugal cmendes@math.uminho.pt 